\title{
Proceedings of the First Probiotics Summit (PBS-2015)
}

\section{$\underline{\text { Keynote Abstracts }}$}

\section{Development of a Live Biotherapeutic Product as a Vaginally Disintegrating Tablet to Prevent HIV Infection}

\author{
Laurel A. Lagenaur ${ }^{\varpi, \AA}$, Thomas P. Parks and Peter P. Lee \\ Osel Inc., USA
}

\begin{abstract}
Women worldwide continue to be infected with HIV at an alarming rate. The majority of Human Immunodeficiency Virus (HIV) infections occur by the mucosal route during unprotected vaginal sexual intercourse. Several commensal Lactobacillus species generally dominate the vaginal microbiota of healthy women of childbearing age and are associated with vaginal health and a reduced risk of bacterial vaginosis (BV), human immunodeficiency virus (HIV), and other sexually transmitted infections (STIs), including herpes simplex virus-2 (HSV-2), and Neisseria gonorrhoeae (gonorrhea). These lactobacilli produce hydrogen peroxide and high levels of D-lactate/lactic acid, which contribute to the maintenance of a low vaginal $\mathrm{pH}(4.0-4.5)$ and inhibition of genital pathogens.

Osel has bioengineered vaginal lactobacilli to secrete potent HIV inhibitors. These recombinant bacteria can persist on the mucosal surfaces where HIV is transmitted and represent an ideal platform for the development of novel woman-controlled and coital-independent biotherapeutics. In a recent proof of concept study our recombinant lactobacillus prevented simian (S)HIV infection in a macaque vaginal challenge model. The lactobacillus product (MucoCept) has been formulated as a tablet, which is stable for over a year at $4-25^{\circ} \mathrm{C}$. MucoCept is being developed as an Investigational New Drug/Live Biotherapeutic Product (LBP) to prevent HIV infection in women.
\end{abstract}

\section{Role of Microbiota-gut-brain Axis in Mental Health with the Emphasize of Auto- antibodies: Effect of Probiotics and Nutritional Interactions}

\author{
Afaf El-Ansary ${ }^{\square, i}$ and Laila Al-Ayadhi \\ King Saud University, Saudi Arabia
}

\begin{abstract}
Microbial colonization of mammals is an evolution-driven process that modulates host physiology, many of which are associated with immunity and nutrient intake. The gut microbiota is increasingly recognized as a complex signaling network that impacts on many systems beyond the enteric system modulating, among others, cognitive functions including learning, memory and decision-making processes. This has led to the concept of a microbiota-driven gut-brain axis, reflecting a bidirectional interaction between the brain and the gut. Immune mechanisms that maintain intestinal homeostasis tend to minimize contact of intestinal bacteria with the gut epithelium. Gut microbiota can elicit signals via the vagal nerve to the brain and vice versa. Modulation of transmitters (e.g., serotonin, melatonin, gamma-aminobutyric acid, histamines, and acetylcholine) within the gut is yet another possible mechanism of action that could mediate the effects of the gut microbiota on mental health. Recently new data further validates a role for molecular mimicry and auto-antibodies in mediating the gut-brain axis and subsequently participating in the development of depression and neurodevelopmental disorders, such as autism and schizophrenia. The relationship between diet and the gut microbiota progresses and became more meaningful. Microbiota-dependent response of
\end{abstract}


animals to diet and nutritional interactions between different dietary regimes and gut microbiota is an interesting phenomenon. Understanding the pathophysiologic mechanisms involving the bidirectional brain-gut axis signaling and the impact of alteration of gut-microbiota on functional and behavioral changes in animals as well as in clinical studies could help to suggest that modulation of the gut-microbiota by therapeutic (e.g. probiotic and pre-biotic) agents opens a new promising strategy for brain-related disorders.

\title{
Rethinking Probiotics and Nutraceuticals: The Health Paradigm
}

\author{
Antonello Santini ${ }^{\bullet}$ \\ University of Napoli Federico II, Italy
}

\begin{abstract}
Probiotics, living microorganisms which are considered to promote good health, are extensively studied with the aim of a continuous and progressive unravelling of plant and bacterial effects on systemic immune and this has led to new credence for the use of probiotics and nutraceuticals in clinical medicine. Nutraceuticals are defined by the Merriam-Webster dictionary as "a food that provides benefits health in addition to its nutritional content". The term refers to active substances extracted from plants, concentrated and administered in a pharmaceutical suitable form. It is worldwide recognized that diet and lifestyle are essential to promote and maintain the condition of well-being and nicely-being and prevent disease onset. Scientific evidences have been acquired for the use of probiotics in other gastrointestinal infections, irritable bowel syndrome and inflammatory bowel disease. The use of nutraceuticals and probiotics as therapeutic agents for gastrointestinal disorders and other different pathologic conditions is rapidly moving into clinical usage. Scientific studies are providing mechanisms of action to explain the therapeutic effects, and randomized controlled trials are providing the necessary evidence for their incorporation into the therapeutic armamentarium. Lactic acid bacteria and bifidobacteria are examples of probiotics. They are found in products such as yogurt to which cultures are added as part of the fermentation process. Once in the intestinal tract, they flourish to the point where potentially harmful bacteria that come along cannot grow due to the competition from these beneficial microorganisms. Metabolic syndrome, a worldwide epidemic disease, is an example of association existing between health conditions onset and wrong lifestyle and dietary habit. This syndrome is characterized by cardio metabolic risk factors that include obesity, insulin resistance, hypertension, and dyslipidemia. Nutraceuticals, generally recognized as safe, and probiotics with proven clinical efficacy can be a promising tool to prevent and support the cure of some pathologic conditions and they can be used in the range "beyond the diet, before drugs".
\end{abstract}

\section{Highly Efficient Extraction Method for Gas Chromatographic Analysis of Conjugated Linoleic Acid (CLA) from Probiotic Bacteria Grown MRM Medium:A Rapid Screening Tool for the Probiotic Bacteria Having CLA Producing Activity}

\author{
Mun YhungJung \\ Woosuk University, Republic of Korea
}

\begin{abstract}
Efficient extraction method for gas chromatographic analysis of CLA from probiotic bacteria grown MRM medium was developed for the rapid screening of probiotics having high CLA producing activity. Different solvents were test for the recovery of CLA from probiotic bacteria grown in de Man, Rogosa, and Sharpe medium. As compared with chloroform/methanol and hexane/isopropanol, hexane provided high extraction efficiency for CLA from unspent de Man, Rogosa, and Sharpe medium. The extraction of oleic acid originated from the emulsifier in broth was also greatly influenced by the type of solvents. The $\mathrm{pH}$ of the system also greatly affect the extraction efficiency of CLA, showing the optimal $\mathrm{pH}$ of 7.0. Repeated extraction with hexane increased the CLA extraction yield, reaching up to 97.2\%. This represents the highest recovery of CLA from cultured broth ever reported. The sample size can be successfully reduced to $0.5 \mathrm{~mL}$ to analyze CLA from the broth. This reduced sample size in the 1.5-mL microcentrifuge tube using a small bench-top centrifuge greatly reduced analytical time. With this analytical method, probiotic bacteria having high CLA producing activity were successfully screened.
\end{abstract}




\title{
$\underline{\text { Speaker Abstracts }}$
}

\section{Technological Characteristics of Probiotics in Traditional Fermented Beverages}

\author{
Dilek Heperkan ${ }^{\square}$ \\ Istanbul Technical University, Turkey
}

\begin{abstract}
Probiotics are living bacteria obtained from different sources. Probiotics are available as dietary supplements or they may be incorporated directly into foods. Fermented food products are important in human diet all around the world due to their health benefits. In addition, they can play an important role as vehicle to carry alive bacteria since most of them are consumed without cooking. Actually, their health benefits are partially coming from probiotics. Traditional fermented non-alcoholic beverages have been taking great attention from the researchers and consumers recently due to their probiotic potentials. Shalgam juice, hardaliye, boza, ayran and kefir are well-known traditional Turkish fermented drinks. Among them, especially boza which is produced by boiling coarsely ground cereals in water are considered to be the most important product in terms of health benefit since it is consumed by all age groups. However, probiotics may show poor viability in fermented products due to detrimental conditions. The number of probiotic lactic acid bacteria often decline during transportation and storage. In this presentation the effect of factors such as microbial competition, metabolites and antimicrobial metabolites of microbial population will be discussed. Some technological characteristics including antibacterial properties of possible probiotic bacteria in some traditional Turkish fermented non-alcoholic beverages will also be presented.
\end{abstract}

\section{Properties of Leuconostoc mesenteroides subsp. mesenteroides SJRP55: A Multitasking Strain}

\author{
Ana Lucia Barretto Penna ${ }^{1, \varpi,\llcorner, ~ A l i n e ~ T e o d o r o ~ d e ~ P a u l a ~}{ }^{1}$, Luana Faria Silva ${ }^{1}$ and Svetoslav Dimitrov Todorov ${ }^{2}$ \\ ${ }^{1}$ UNESP - Sao Paulo State University, Brazil \\ ${ }^{2}$ Federal University of Viçosa, Brazil
}

\begin{abstract}
Leuconostoc mesenteroides subsp. mesenteroides SJRP55 was isolated from water-buffalo mozzarella cheese, and was identified based on the $16 \mathrm{~S}$ rRNA gene sequencing. The present study focus on the characterization of the production of antimicrobial compounds, probiotic potential, and technological properties of Ln. mesenteroides subsp. mesenteroides SJRP55. The strain produced antibacterial compounds against Listeria spp. strains and did not inhibit Lactobacillus spp. The antimicrobial substances were stable at high temperatures and low $\mathrm{pH}$ values, but sensitive to proteolytic enzymes and resistant to $\alpha$-amylase, lipase, and catalase enzymes. The strain produced at least 2 different active compounds that exhibited a bacteriostatic mode of action against different indicator microorganisms, including Listeria spp. The bacteriocins were identical to mesentericin Y105 and B105. For the probiotic characterization, the microorganism presented resistance to stressful conditions that simulated the gastrointestinal tract, ability to deconjugate bile salts, good adhesion properties, $\beta$-galactosidase activity, and sensibility to most of the tested antibiotics. The DNA analysis revealed presence of open reading frames possibly coding for virulence factors; however, biogenic amines encoding genes were not observed. Considering the technological characterization, Ln. mesenteroides subsp. mesenteroides SJRP55 presented the ability to utilize citrate, showed proteolytic activity, was able to reduce the $\mathrm{pH}$ to below 5.0 and to produce high concentration of organic compounds. The novel indigenous Ln. mesenteroides subsp. mesenteroides SJRP55 may be used as a bio-preservative culture in the dairy industry and as a potential probiotic strain, and besides it provides desirable aroma and flavor to fermented products.
\end{abstract}

\section{New Potential Probiotic Strain of Leuconostoc citreum with Immunomodulatory Effects}

\author{
Celia Costa Gomes da Silva ${ }^{1, \varpi,\llcorner, ~ M a r i n a ~ F . ~ P . ~ D o m i n g o s ~ L o p e s ~}{ }^{1}$, Catherine Stanton ${ }^{2}$ and Paul R. Ross ${ }^{2}$ \\ ${ }^{1}$ CITAA/Universidade dos Açores, Portugal \\ ${ }^{2}$ Teagasc Moorepark Food Reseach Centre, Ireland
}




\begin{abstract}
Allergies have become increasingly prevalent during the past few decades, resulting in a growing interest on the use of probiotics as possible anti-allergic therapy. The present study was aimed to primarily assess the immunomodulating properties of a strain of Leuconostoc citreum (L3C1E7), isolated form an artisanal cheese. This strain was found to tolerate gastrointestinal digestion, adhere to colon (Caco-2) cells and produce large amounts of exopolysaccharide. Results from in vitro tests showed that the live strain had a significant effect on reducing the pro-inflammatory cytokine IL-8 release by lipopolysaccharideinduced HT-29 cells. In addition, a rat model was used to demonstrate any beneficial probiotic effects. Rats fed with ovalbumin (OVA), with/without addition of Ln. citreum L3C1E7, were vaccinated subcutaneously with OVA on day 7 and 14 . It was observed that OVA specific IgG1 and IgG2a responses were restored after pre-feeding with this strain. In a second experiment, an OVA-induced asthma model was used. Rats were fed OVA in conjunction with skin immunization and nasal sensitization with OVA. In this experiment, down-regulated IgG1 and IgG2a responses to OVA were enhanced after nasal sensitization with OVA, but were restored in rats that were pre-feed with $L n$. citreum L3C1E7. By contrast, OVA specific gut IgA responses were enhanced when Ln. citreum was used as oral adjuvant. In addition, total IgE in plasma was significantly reduced by pre-feeding with Ln. citreum L3C1E7. These data show that Ln. citreum L3C1E7 suppresses specific IgE synthesis and may alleviate allergic symptoms in animals.
\end{abstract}

\title{
Chemical Sensing in the Colon
}

\author{
Atsukazu Kuwahara" \\ University of Shizuoka, Japan
}

\begin{abstract}
The gastrointestinal (GI) tract is separated from the body's internal environment by a single layer of epithelial cells, through which nutrients must pass for their absorption into the blood stream. Approximately 100 trillion bacteria, which are termed the gut microbiota, are present in the intestinal lumen, especially in the colon. The genome of the gut microbiota contains an estimated 150 times as many genes as in the host genome, and continuously produces large amount of various chemicals, including SCFAs, which can be beneficial or harmful to the host. Therefore, the GI tract has to monitor the composition of its contents continuously to discriminate between necessary and unnecessary compounds. These responses occur at the level of the mucosa, which contains epithelial cells. Recent molecular identification of epithelial membrane receptor proteins has revealed the sensory roles of intestinal epithelial cells in the gut chemosensory system. The failure of functional interactions within the gut chemosensory system between the host and microbiota may cause a spectrum of diseases beyond local GI disorders, such as obesity, diabetes, metabolic syndrome, and various neurological diseases. Recent studies suggest that SCFAs act as signaling molecules and influence the host's metabolism; uncovering the sensory mechanisms of such bacterial metabolites would help us understand the interactions between the host and microbiota in host energy homeostasis. We will provide an overview of gut chemosensory system on the interaction between microbiota and host, with a particular emphasis on the colon.
\end{abstract}

\section{Black Gram (Phaseolus mungo) Husk as a Potential Prebiotic Enhances Synbiotic Attributes of Yogurt}

\author{
Sarvamangala R. Patil ${ }^{1, \varpi,\llcorner}$ and Dayanand Agsar ${ }^{2}$ \\ ${ }^{1}$ Vishweshwariah College of Applied Sciences, India \\ ${ }^{2}$ Gulbarga University, India
}

\begin{abstract}
Synbiotic is a supplement that includes both a prebiotic and a probiotic which work together to improve the friendly flora of the human intestine. Yogurt and kefir are normally considered as true synbiotic products, since they supply live bacteria and the food they need to survive. Prebiotics are typically non digestible food ingredients that are resistant to human digestive enzymes but serve as food for probiotics. Probiotics are live bacteria which are intended to colonize the large intestine and confer physiological health benefits to the host. Black gram (Phaseolus mungo) is one of the important pulses that are being grown all over India, corresponding to the generation of huge amount of husk. The husk is rich source of dietary fiber and mainly oligosaccharides, which function as prebiotics by increasing levels of beneficial bacteria.

The present investigation was focused on exploration of black gram husk as potential prebiotic and to enrich synbiotic attributes of yogurt. Black gram husk was supplemented to probiotic yogurt containing Lactobacillus casei (NCIM 2364) and
\end{abstract}


Lactobacillus fermentum (NCIM 2165). Microbial, physico-chemical and sensory attributes of probiotics and synbiotics were investigated. The $\mathrm{pH}$ of yogurt was decreased with increase in concentration of husk. The sensitivity to syneresis was significantly higher in case of synbiotic yogurt than probiotic yogurt. The overall sensory quality of yogurt was superior at $1 \%$ concentration of the husk. Therefore, husk of black gram could be an important prebiotic and proved to enhance the high quality attributes of synbiotic yogurt.

\title{
Selection of Probiotic \& Prebiotic and Its Concentration Optimization for the Development of Synbiotic Soy Yoghurt
}

\author{
Shalini Mishra ${ }^{\infty}$ ¿ and H. N. Mishra \\ Indian Institute of Technology, India
}

\begin{abstract}
Preparation of a yogurt-like product from non-dairy raw material such as soy with probiotic and prebiotics is a novel development in the field of fermented functional foods. This research work aimed at finding a new probiotic starter culture and prebiotic for the preparation of synbiotic soy yoghurt which can give good product characteristics and enhance consumer acceptability for fermented soy milk. Various combinations of probiotics St. thermophilus-L. acidophilus, St. thermophilus-L. plantarum, St. thermophilus-L. rhamnosus, St. thermophilus-L. bulgaricus, L. acidophilus-L. plantarum, L. acidophilus - L. rhamnosus and L. plantarum-L. rhamnosus were studied to find the most suitable starter culture. Prebiotics, fructooligosaccharide (FOS) and inulin were added in an attempt to reduce the after-taste of soymilk, improve acidification rates and growth of probiotics. Addition of prebiotic in soymilk (TS $13.23 \%$, TSS $12.5^{\circ} \mathrm{Bx}$, Protein 5.15\%, Fat 2.13\% and pH 6.45) significantly accelerated the acidification rate $\left(10.82\right.$ to $23.33 \times 10^{-3} \mathrm{pH}$ units/min) and reduced the fermentation time by $4-9 \mathrm{~h}$. Sensory analysis reflected that among all studied samples, St. thermophilus-L. acidophilus (ST-LA) fermented FOS supplemented soy milk got highest overall acceptability scores (7.4) on 9 point hedonic scale. Optimized synbiotic soy yoghurt was prepared using FOS (8.1\% $\mathrm{w} / \mathrm{v}$ ) and culture combination ST-LA with inoculum size $3.6 \% \mathrm{v} / \mathrm{v}, 1: 1$ ratio and incubated at temperature $41^{\circ} \mathrm{C}$ for $5.25 \mathrm{~h}$. The developed product showed good nutritional, textual and sensory characteristics. The product was well set (hardness $423.1 \mathrm{~g}$, adhesiveness -111.9 g.s, complex modulus $\mathrm{G}^{*} 1554.3 \mathrm{~Pa}$ ) with very less whey separation (1.14\%). Probiotic count at the end of the $28^{\text {th }}$ day was higher than $109 \mathrm{cfu} / \mathrm{ml}$, which is one of the prerequisites for the probiotic functional foods.
\end{abstract}

\section{Probiotic Bifidobacteria Stimulate SLC26A3 Gene Function and Expression in Intestinal Epithelial Cells}

\author{
Pradeep K. Dudeja ${ }^{\square, \varkappa}$, Anoop Kumar, Cameron Hecht, Shubha Priyamvada, Arivarasu N. Anbazhagan, Anas Alakkam, \\ Alip Borthakur, Ravinder K. Gill and Waddah A. Alrefai
}

University of Illinois at Chicago, USA

\begin{abstract}
SLC26A3 or DRA (Down Regulated in Adenoma) is a key transporter mediating $\mathrm{Cl}^{-}$absorption in intestine. Down regulation of DRA has been implicated in diarrhea associated with inflammatory bowel diseases or infection by food-borne pathogens. Up regulation of DRA, therefore, appears to be a novel approach to treat IBD or infectious diarrhea. Our previous studies showed that increase in DRA function and expression by Lactobacillus acidophilus and its culture supernatant (CS) might underlie anti-diarrheal effects of this probiotic strain. However the effects of Bifidobacterium species, important inhabitants of human colon, on intestinal $\mathrm{Cl}^{-} / \mathrm{HCO}_{3}{ }^{-}$exchange activity were not known. Therefore, studies were carried out to examine the effects of bacteria or their CS on DRA function and expression. CS from B. breve, B. infantis or B. bifidum increased the anion exchange activity ( 2 fold) in Caco-2 cells. Consistent with the function, CS also increased the protein as well as mRNA levels of DRA (but not PAT-1). CS of all three species of Bifidobacteria increased DRA promoter activity $(-1183 /+114 \mathrm{bp})$ in Caco-2 cells ( 2 fold). Further, the increase in DRA mRNA expression by CS of $B$. breve and B. infantis was blocked by transcription inhibitor, Actinomycin D $(5 \mu \mathrm{M})$ and ERK1/2 MAPK pathway inhibitor (U0126, $10 \mu \mathrm{M})$. Administration of live $B$. breve, $B$. infantis and B. bifidum by oral gavage to mice for $24 \mathrm{~h}$ increased the DRA mRNA and protein levels in the colon. These data demonstrate an upregulation of DRA via activation of ERK1/2 pathway that may underlie potential anti-diarrheal effects of Bifidobacterium species.
\end{abstract}




\title{
Lactobacillus plantarum ST8SH as Probiotic or Bio-protective Culture - Safety Aspects for Application
}

\author{
Svetoslav Dimirov'Todorov ${ }^{\varpi, \varkappa}$, Luana Perin and Luis Augusto Nero \\ Universidade Federal de Vicosa, Brazil
}

\begin{abstract}
Lactobacillus plantarum ST8Sh was isolated from Bulgarian salami and been identified based on his biochemical and genetic features including PCR with Species-specific primers and 16S rRNA sequencing. This strain produces class IIa bacteriocin $(5.0 \mathrm{kDa})$, active against different species from genera Enterobacter, Enterococcus, Lactobacillus, Pseudomonas, Streptococcus and Staphylococcus and different strains of Listeria spp. including more than 80 different L. monocytogenes strains from various serological groups. Based on the PCR analysis, Lb. plantarum ST8Sh was hosting gene related to the production of variant of pediocin PA-1. In addition, $L b$. plantarum ST8Sh harbors genes related to the production adhesion proteins, such as Mab, Mub, EF, PrgB. Based on the genetic and physiological tests, Lb. plantarum ST8Sh presented a potential to be consider as a probiotic candidate. Total DNA extracted from $\mathrm{Lb}$. plantarum ST8Sh have been screened for presence of more than 50 genes related to production of biogenic amines (histidine decarboxylase, tyrosine decarboxylase and ornithine decarboxylase), virulence factors (sex pheromones, gelatinase, cytolisin, hyaluronidase, aggregation substance, enterococcal surface protein, endocarditis antigen, adhesion of collagen, integration factors) and antibiotic resistance (vancomycin, tetracycline, erythromycin, gentamicin, chloramphenicol, bacitracin). Lb. plantarum ST8Sh showed a low presence of virulence genes. Only 13 genes were detected (related to sex pheromones, aggregation substance, adhesion of collagen, tetracycline, gentamicin, chloramphenicol, erythromycin, but not to vancomycin and bacitracin) and may be considered as safe for application in fermented food products. Besides all beneficial properties studied for various LAB, most considered as GRAS, a special attention need to be addressed on the possible presence of virulence factors, production of biogenic amines and antibiotic resistance. These virulence determinants have been detected and well-studied in Enterococci and Streptococci, however, in last few years report on presence of virulence factors in otherwise GRAS Lactobacilli have been showing the potential upcoming problems. Horizontal gene transfer of virulence factors between pathogenic and LAB, including probiotics, is a highly possible scenario in case of uncontrolled application of probiotics.
\end{abstract}

\section{Gut Inflammation and Altered Microbiome Impact Progression of Chronic Kidney Disease}

\author{
Wei Ling Lau $\square$ \\ University of California, USA
}

\begin{abstract}
Chronic inflammation is a non-traditional risk factor for cardiovascular mortality in the chronic kidney disease (CKD) population. In recent years the gastrointestinal tract has emerged as a major source of systemic inflammation in CKD. Uremic toxins induce gut wall inflammation, and the subsequent breakdown of the epithelial tight junction barrier results in a "leaky gut" with translocation of bacterial toxins into the bloodstream. Further, dietary restrictions in CKD patients leads to alteration of the normal gut microbiome, leading to overgrowth of bacteria that produce uremic toxins, and translocation of these toxins into the bloodstream further promotes systemic inflammation. Trials are ongoing to study prebiotic and probiotic interventions on clinical outcomes in the CKD population.
\end{abstract}

\section{Helicobacter pylori and Gastric Cancer: Indian Enigma}

\author{
Renu Pandey ${ }^{1, \sqsubset,\llcorner, ~ V a t s a l a ~ M i s r a ~}{ }^{2}$, S. P Misra ${ }^{2}$ and Manisha Dwivedi ${ }^{2}$ \\ ${ }^{1}$ SDSMT Rapid City South Dakota, USA \\ ${ }^{2} M L N$ Medical College, India
}

\begin{abstract}
Introduction: Helicobacter pylori is classified as class 1 carcinogen. In India and various other countries a difference in prevalence of $H$. pylori infection and gastric cancer has been noted. This puzzle of $H$. pylori distribution and gastric cancer
\end{abstract}


epidemiology is termed as Indian enigma.The present study aims to find the correlation between $H$. pylori infection and the progression of carcinogenesis, by studying helicobacter pylori infection in north Indian population, its relationship with apoptosis and p53 gene in gastric biopsies.

Material and Method: 921 biopsies were collected and tested for prevalence of $H$. pylori by RUT, Imprint cytology and Histology. Apoptosis was studied by TUNEL method. Analysis of $\mathrm{p} 53$ gene polymorphism at codon 72 was done by PCR using restriction enzyme BstU1.

Observation: Out of 921 samples tested 56.73\% (543) were H. pylori Positive by three techniques RUT, Imprint cytology and Histology. The mean Apoptotic Index (AI) in normal group was 2.12. Gastritis had the maximum AI (4.24) followed by GU (2.28), GP (2.22) and DU (2.08). Mean AI in cases with gastric cancer was less than the normal group (1.72). The analysis of p53 72 SNP reveal that p53 (Arg/Arg), (Pro/Arg) variant are higher (40.59\% \& 33.66\%) as compared to p53 pro/pro variant $(25.74 \%)$ in healthy population.

Conclusion: There was slightly higher prevalence of $H$. pylori in controls as compared to the patient group as the PUD diseases are less than $5 \%$ so the bacterium cannot be only factor responsible for development of disease.

\title{
Role of Antioxidants and Polyphenols in Thrombosis and Haemostatic Function
}

\author{
Abishek B. Santhakumar ${ }^{1, \sqsubset, ~}$, Avinash R. Kundur ${ }^{2}$, Kent Fanning ${ }^{3}$, Michael Netzel ${ }^{4}$, Natalie Colson ${ }^{2}$, Roger Stanley $^{5}$ and \\ Indu Singh ${ }^{2}$ \\ ${ }^{1}$ Central Queensland University, Australia \\ ${ }^{2}$ Griffith University, Australia \\ ${ }^{3}$ Agri-Science Queensland, Australia \\ ${ }^{4}$ The University of Queensland, Australia \\ ${ }^{5}$ University of Tasmania, Australia
}

\begin{abstract}
Platelet hyperactivity and oxidative stress play a central role in the pathogenesis of many disorders including cardiovascular disease, thrombosis and type 2 diabetes. Natural antioxidants and polyphenols are believed to exhibit cardio-protective properties via their free radical scavenging activity. In this research, the anti-thrombotic potential of taurine, caffeine, anthocyanin polyphenol and its active metabolite hippuric acid, was evaluated. It was found that taurine and caffeine, in vitro, synergistically lowered platelet aggregation and prolonged time taken for clot formation. Dietary supplementation with a novel variety of Queen Garnet plum juice (QGPJ), rich in anthocyanins, for 4 weeks reduced platelet hyperactivity/aggregation, reduced biomarkers of oxidative stress, and favorably altered coagulation parameters in normal healthy individuals. This antithrombotic activity of QGPJ was greater under an exercise-induced model of oxidative stress in healthy individuals. Hippuric acid, in vitro, also demonstrated reduction of platelet hyperactivation. Supplementation with QGPJ did not alter thrombosis specific gene expression. The results demonstrate the potential of natural antioxidants in reducing platelet hyper activation related thrombogenesis in both normal and prothrombotic conditions. Further mechanistic studies are warranted to unveil the potential of such natural compounds in complementary antiplatelet therapy.
\end{abstract}

\section{Isolation of Very Tolerant Probiotics for Food Products with Harsh Conditions}

\author{
Amir M. Mortazavian ${ }^{\varpi, \AA}$ and Sara Sohrabvandi \\ Shahid Beheshti University of Medical Sciences, Iran
}

\begin{abstract}
Nutraceutical effectiveness of probiotics in foods is determined by the rate of probiotics viability in final products at the time of consumption. The amount of $107 \mathrm{cfu} \mathrm{m}^{-1}$ is recognized as the minimum level. The main vegetative (non-spore formers) probiotics used in foods belongs to genera Bifidobacterium and Lactobacillus. The greatest drawback of food probiotic technology is viability loss of probiotic cells during processing and particularly, during the storage time due to their susceptibility to the harsh conditions of products. $\mathrm{pH}$ and acidity, molecular oxygen, and time and temperature of storage are the most detrimental factors. Furthermore, if a high number of viable cells have been protected in products by any method, it is not guaranteed that the same numbers would be reached to the intestine owing to the exposing the cells to very harsh in vivo conditions. Although much has been investigated and published about improvements on food formulations and processes in order to make them more adjustable to probiotic strains nature, practically and economically, the best way is to use tolerant strains. We have isolated
\end{abstract}


and modified numerous Iranian native probiotic strains with outstanding resistance to very harsh conditions of fermented milk drinks, fruit juices and beer. Recently, these strains are industrially manufactured and sold to dairies in Iran and are being marketed in other countries. In this article, the resistance characteristics of mentioned probiotic strains (bifidobacteria and lactobacilli) to foods and simulated GIT are discussed. This new generation of probiotic strains can evolve the food probiotic technology.

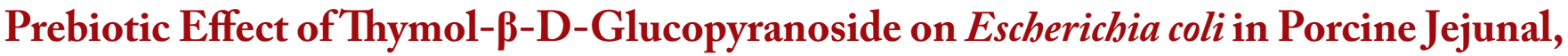 Cecal and Rectal gut Compartments
}

\author{
Gizem Levent $^{1,2}$, Gurhan Ciftcioglu ${ }^{2, \varpi, \varkappa}$, Robin C. Anderson ${ }^{1}$, Ross C. Beier $^{1}$ and David J. Nisbet $^{1}$ \\ ${ }^{1}$ United States Department of Agriculture, USA \\ ${ }^{2}$ Istanbul University, Turkey
}

\begin{abstract}
A prebiotic is a selectively fermented ingredient that allows specific changes, both in the composition and/or activity in the gastrointestinal microflora that confers benefits upon host well being and health. Antimicrobial activity of certain compounds may be considered as prebiotic affect, when they show their activity, for the benefit of probiotic bacteria. Thymol is a natural antimicrobial with activity against zoonotic pathogens but its rapid absorption in the proximal gut prevents its passage to the lower gut where these pathogens primarily reside. Thymol- $\beta$-d-glucopyranoside ( $\beta$-thymol) passes more effectively than free thymol to the lower gut but needs to be hydrolyzed by $\beta$-glycoside-hydrolyzing bacteria in order to liberate free thymol. Anti-Escherichia coli ( $E$. coli) activity of $\beta$-Thymol has not been studied, where various $\beta$-glycoside-hydrolyzing gut microbes would be present. This study aimed to analyze the presence of $\beta$-thymol hydrolyzing-activity in digesta collected from various gut compartments of a weaned pig and as evidenced by the anti-E. coli effect of free thymol potentially liberated by these populations. Jejunal, cecal and rectal contents were added (6,15 and $15 \mathrm{~g}$ respectively) to separate $100 \mathrm{~mL}$ volumes of anaerobic Mueller Hinton broth inoculated with $5.76 \pm 0.1 \log 10 \mathrm{CFU} / \mathrm{mL}$ E. coli K88, which is a virulent zoonotic pathogen causing economically important disease in young swine. The suspensions were distributed to crimp top tubes (10 mL/tube) supplied with or without $6 \mathrm{mM} \beta$-thymol and incubated anaerobically under $100 \% \mathrm{~N} 2$ at $39^{\circ} \mathrm{C}$. Survivability of E. coli was determined by plating serial dilutions on MacConkey Agar supplemented with $25 \mu \mathrm{g} / \mathrm{mL}$ nalidixic acid and $20 \mu \mathrm{g} / \mathrm{mL}$ novobiocin and viable colonies counted after $24 \mathrm{~h}$ incubation $\left(37^{\circ} \mathrm{C}\right)$. After $6 \mathrm{~h}$ incubation, viable $E$. coli in cecal and rectal suspensions were decreased $(\mathrm{P}<0.05)$ from those in controls $(6.3 \pm 0.4$ and $6.2 \pm 0.2 \log 10 \mathrm{CFU} / \mathrm{mL}$, respectively) by $6 \mathrm{mM}$; and $2.3 \log 10$ and $2.2 \log 10 \mathrm{CFU} / \mathrm{mL}$ by $\beta$-thymol-treatment in cecal and rectal suspensions, respectively. Similarly, $E$. coli counts in $\beta$-thymoltreated cecal and rectal suspensions were decreased 2.1 and $0.5 \log 10 \mathrm{CFU} / \mathrm{mL}$ from control counts $(5.7 \pm 0.05$ and $4.9 \pm 0.2$ $\log 10 \mathrm{CFU} / \mathrm{mL}$, respectively) after $24 \mathrm{~h}$ incubation. In jejunal suspensions, control and treated E.coli counts differed by only $0.45 \log 10 \mathrm{CFU} / \mathrm{mL}$ after $6 \mathrm{~h}(8.84 \log 10 \mathrm{CFU} / \mathrm{mL}$ and $8.39 \log 10 \mathrm{CFU} / \mathrm{mL}$, respectively) but were decreased $(\mathrm{P}<0.05)$ from controls $(7.14 \pm 0.02 \log 10 \mathrm{CFU} / \mathrm{mL})$ by $2.31 \log 10$ by $\beta$-thymol after $24 \mathrm{~h}$. Results reveal an anti-E. coli effect of $\beta$-thymol in microbial populations from various porcine gut compartments, and therefore a prebiotic affect, which yields probiotics to dominate. However, more studies are needed to be understood the kinetics of $\beta$-thymol activity in swine gut.
\end{abstract}

\section{Probiotic Strains for Value Addition in Tropical Fruits}

\author{
Puja Ravikumar ${ }^{\varpi, ¿, ~ R a v i s h ~ B h a t, ~ L a t h a ~ D i w a k a r ~ a n d ~ A n i l ~ K u s h ~}$ \\ Vittal Mallya Scientific Research Foundation, India
}

\begin{abstract}
Common tropical fruits like guava and papaya are nutrient rich and available almost all through the year. Post harvest losses in these fruits are enormous due short shelf life and susceptibility to fungus induced rapid decay. We have used bacterial fermentation for value addition and to address the post harvest losses of these abundantly available fruits. Lactobacillus plantarum mediated 25 hours fermentation of raw guava pulp improved the total phenolic content, anti-oxidant potential and short and medium chain fatty acids. Probiotics mediated fermentation of raw papaya enhanced the anti oxidant content and the fermented product is amenable for blending in a variety of food items. In an alternate approach, bacterial endophytes from ripe papaya fruits were isolated, characterized and used in fermentation cocktails along with Saccharomyces cerevisiae. Fermentation was terminated after $21 \mathrm{~h}$ and the lyophilized product was an off white water soluble amorphous powder. Our bio-efficacy studies on mice model indicate a positive immunomodulatary effect of the fermented product. The fermented product, as filtrate or lyophilized powder can be used for developing an array of nutritionally enhanced products like bars and drinks.
\end{abstract}




\title{
Longer Period of Oral Administration of Aspartame on Cytokine Response in Wistar Albino Rats
}

\author{
Arbind Kumar Choudhary ${ }^{\square, \AA}$ and Rathinasamy Sheela Devi
}

University of Madras, India

\begin{abstract}
Introduction: Aspartame is a non-nutritive sweetener particularly used in 'diet' and 'low calorie' products and also in a variety of foods, drugs and hygiene products. Aspartame is metabolized by gut esterases and peptidases to three common chemicals: the amino acids, aspartic acid and phenylalanine, and small amounts of methanol. The aim of the present study was to assess potential changes in molecular mediators of aspartame as a chemical stressor in rats.
\end{abstract}

Materials and Methods: The effects of long-term administration of aspartame (40 mg/kg bodyweight/day) were tested in Wistar Albino rats. The treatment effects were assessed in different conditions, including control groups. After 90 days of treatment, circulating concentrations of different parameters were assessed: corticosterone, lipid peroxidation, antioxidant activity, nitric oxide, reduced glutathione and cytokines (interleukin 2, interleukin 4, tumor necrosis factor- $\alpha$ and interferon- $\gamma$ ).

Results: The results show that there was a significant increase in plasma corticosterone, serum lipid peroxidation and nitric oxide level along with a decrease in enzymatic and non-enzymatic antioxidant as well as significant decrease in interleukin 2 , tumor necrosis factor- $\alpha$ and interferon- $\gamma$. There was also a significant increase in interleukin 4 irrespective of whether the animals were immunized or not.

Conclusion: The findings clearly point out that aspartame acts as a chemical stressor because of increased corticosterone level and increased lipid peroxidation and nitric oxide level induce generation of free radicals in serum which may be the reason for variation of cytokine level and finally results in alteration of immune function. Aspartame metabolite methanol or formaldehyde may be the causative factors behind the changes observed.

\section{Effect of Processing on the Functionality of Thylakoid Membranes as an Appetite Suppressing Agent}

\author{
Karolina Ostbring ${ }^{1, \varpi,\llcorner}$, Ingegerd Sjoholm ${ }^{2}$, Charlotte Erlanson-Albertsson ${ }^{1}$ and Marilyn Rayner ${ }^{2}$ \\ ${ }^{1}$ Institution of Experimental Medical Science, Lund University, Sweden \\ ${ }^{2}$ Institution of Food Engineering, Lund University, Sweden
}

\begin{abstract}
The western diet contains high proportions of palatable food, rich in refined carbohydrates and fat, which subsequently increases the risk of overconsumption and possibly lead to overweight and obesity. Thylakoid membranes isolated from green leaves have been found to increase satiety by prolonging lipolysis. Administration of thylakoids to animals and humans reduced food intake and body weight as well as affected appetite-regulation hormones. However, the effect of industrial processing as heat treatment, different drying techniques and storage of thylakoids has not yet been previously evaluated. Heat treatment reduced the ability to inhibit lipase/co-lipase in vitro, thus a reduced functionality of heat-treated thylakoid membranes. Drum drying, spray drying and freeze-drying were investigated and drying at higher temperatures induced alterations of the interfacial properties of the thylakoids hence lower lipase-inhibiting effect. Freeze-drying, with temperatures not exceeding $25^{\circ} \mathrm{C}$, was therefore concluded as the most suitable drying technique with respect to functionality of the resulting powder. A correlation between characteristics and functionality of the thylakoids was established where chlorophyll content, green colour and lightness of powders were correlated to lipase inhibiting capacity. This opens possibilities to partly replace the enzymatic model with the less time consuming and more cost efficient spectrophotometric method in screening processes. Knowledge about the effect of processing is important to produce a functional food with retained functionality. Thus the results can be applied in production of a more standardised functional food ingredient with optimized appetite reducing properties.
\end{abstract}




\title{
Bacterial Transplantation, a Novel Approach to Evaluate the Efficacy of Probiotic Candidates against Enteric Pathogens in Animals
}

\author{
Komala Arsi $^{1, \varpi, \iota}$, A. M. Donoghue ${ }^{2}$, A. Woo-Ming ${ }^{1}$, P. J. Blore ${ }^{1}$ and D. J. Donoghue ${ }^{1}$ \\ ${ }^{1}$ University of Arkansas, USA \\ ${ }^{2}$ Agricultural Research Service, USDA, USA
}

\begin{abstract}
Campylobacter is the leading cause of foodborne illness worldwide, primarily caused by consumption of contaminated poultry products. One strategy to reduce Campylobacter colonization in poultry is the use of oral probiotics, but results are variable, possibly because the probiotics are destroyed in the stomach's acidic environment. Protection (e.g., encapsulation) of isolates may overcome this problem, but there is no assurance these isolates will have efficacy in the lower gastrointestinal tract. Therefore, screening candidate isolates by directly placing them in the lower intestinal tract via cloacal (rectal) inoculation may eliminate the time and expense of encapsulating ineffective isolates. Thus, the purpose of this study was to collect bacterial isolates with anti-Campylobacter activity in vitro and evaluate their efficacy in vivo upon either oral or intra-cloacal administration. Bacterial isolates were collected from healthy birds and isolates were screened for efficacy against Campylobacter in vitro. Ten isolates demonstrating in vitro anti-Campylobacter properties were evaluated after oral or intra-cloacal inoculation into chicks on day 1 ( $\mathrm{n}=10$ birds/isolate/location). Birds were orally challenged with Campylobacter jejuni at day 7 and ceca were collected on day 14 for Campylobacter enumeration. When dosed orally, only one isolate showed a 1-log reduction in cecal Campylobacter counts, whereas when administered intra-cloacally, six isolates produced a 1-3 log reduction in cecal Campylobacter counts. These results support the strategy of evaluating the efficacy of potential probiotic isolates via cloacal inoculation prior to undergoing encapsulation for oral administration and may provide a strategy for evaluating prospective probiotic isolates in other animal models.
\end{abstract}

\section{Lactobacillus and Enterococcus Strains Isolated from Breast Milk Exhibit Potential Anti- bacterial Activity Against Food-borne Pathogens}

\author{
Po-Wen Chen ${ }^{1, \varpi, \varkappa}$, Mao-Sheng Huang ${ }^{2}$ and Shu-Ying Tseng ${ }^{3}$ \\ ${ }^{1}$ St. Mary's Junior College of Medicine, Republic of China \\ ${ }^{2}$ St. Mary's Hospital Luodong, Taiwan, Republic of China \\ ${ }^{3}$ National Chung-Hsing University, Republic of China
}

\begin{abstract}
Human breast milk is an important nutrient for infant development. The milk is known to contain diverse bacteria including lactic acid bacteria (LAB), the functions of which are not well-understood. Lactoferrin (Lf) is a natural glycoprotein found mainly in milk. This protein possesses broad-spectrum anti-bacterial activities; however, its influence on the growth of milkisolated probiotic bacteria is not clear. In this study, milk samples were collected from 14 mothers between 3 and 360 days postpartum. One LAB species, Lactobacillus gasseri, and one potential LAB species, Enterococcus faecalis were isolated, and their anti-bacterial potential was evaluated in vitro. Supernatants produced by these species exhibited anti-bacterial activity against food-borne pathogens, including Escherichia coli (HER 1255), Salmonella enterica (ATCC 19430), and Staphylococcus aureus (ATCC 25923). The effect of bovine Lf (bLf) on milk-isolated L. gasseri and E. faecalis were also examined. L. gasseri and $E$. faecalis were resistant to the anti-bacterial activity of bLf. In contrast, $\mathrm{bLf}$ inhibited the growth of the silage LAB, L. coryniformis (ATCC 25602) and an E. faecalis strain isolated from urine. Notably, bLf also inhibited the growth of food-borne pathogens, including E. coli, S. enterica, and S. aureus. The bLf MIC for L. gasseri and E. faecalis isolated from milk was 4-8-fold higher than for pathogens. In conclusion, milk-isolated Lactobacillus and Enterococcus exhibit anti-bacterial activity and bLf-resistance. This may partially explain the function of these two species in milk. The present study merits further examination of a practical approach combining milk LAB and bLf in the field.
\end{abstract}

\section{The Potential Possibility of Indonesian 'tempeh' for Its Usage in Marine Aquaculture Products}

\author{
Rahmad Supriyanto ${ }^{\square}$ \\ Istanbul Technical University, Turkey
}




\begin{abstract}
The human consumption of marine aquaculture products are getting increased over the past decades, as it is a good source of protein. As the intensive farming in marine products to meet the demands, the vast usage of antibiotics is used. This has resulted to improper way of farming marine products which leads to several adverse health effects prior consumption and to the cultured species. The use of probiotics in aquaculture products is to reduce the risk of health effects during farming, and as an alternative way to reduce the use of antibiotics. Tempe is one of the fermented soy bean products from Indonesia which has potentiality as probiotics source used in marine aquaculture. This paper shows the potential of using strains from Tempe for production of probiotics in aquaculture, its preceding, and application to be carried out and discusses the prospects for study in this field.
\end{abstract}

\title{
Antioxidant Activity of Diallyl Sulphide Against Toxin-induced Oxidative Stress and Antioxidant Defense System
}

\author{
Sabah Ansarø \\ King Saud University, Saudi Arabia
}

\begin{abstract}
Garlic has been reported to possess therapeutic properties as it contains diallyl sulphide (DAS) and other structurally related compounds which are widely believed to be active agents in preventing cancer. This study investigates the effect of DAS (a phenolic antioxidant used in foods, cosmetics and pharmaceutical products) on mercury-induced hepatotoxicity in rats. Male albino rats of Wistar strain (4-6 weeks old) weighing 180-200 g were used in this study. Animals were given a single dose of $\mathrm{MgCl}_{2}(50 \mathrm{mg} / \mathrm{kg}$ body weight, intraperitoneal) after a week of treatment with DAS - $200 \mathrm{mg} / \mathrm{kg}$ body wt $/ \mathrm{ml}$ corn oil respectively administered through the gavage. $\mathrm{HgCl}_{2}$ administration led to about 2 fold enhancement in the values of both ALT and AST respectively, and the activities of glutathione metabolizing and antioxidant enzymes decreased to a range of 2-2.2 fold. These changes were reversed significantly $(\mathrm{p}<0.001)$ in animals receiving a pretreatment of DAS. DAS afforded protection against hepatic increase in glutathione levels and glutathione metabolizing enzymes to $60-70 \%$ as compared to $\mathrm{HgCl}_{2}$ alone treated group. Also, histological changes in the liver were investigated. The morphological damage in the liver was reduced in DAS pretreated mercuric chloride group as compared to control group.
\end{abstract}

\section{Importance and Impact of Probiotics in Dentistry}

\author{
Erandi Escamilla-Garcia \\ Universidad Autónoma de Nuevo León (UANL), Mexico
}

\begin{abstract}
Tooth decay affects humans of all ages in the world and remains the main problem of oral health among children. It is a disease that can hardly be eradicated because of its complex interplay of social, cultural, nutritional and biological factors that are associated with the initiation and progression of it.

Childhood and adolescence are critical periods in the development of a healthy dentition. Education on oral hygiene from an early age has become a system of routine for dentists, however, tooth-decay and periodontal-diseases remains a significant not only in adults but also in children and adolescents problem. To prevent further destruction of the tooth and promote better health in general there are various treatments.

Lactic acid bacteria (LAB) are well known for their beneficial effects on human health in the intestine and immune system, however, there are few studies on the impact they can generate in oral-health. Lactobacillus reuteri is part of microorganisms used as probiotics in areas such as food and medicine, because it has antimicrobial properties among others.

In vitro studies have demonstrated the effectiveness of $L$. reuteri for the inhibition of oral bacteria such as those present on cariogenic and periodontal-diseases, as: Porphyromonas gingivalis, Fusobacterium nucleatum, Actinomyces actinomycentemcomitans, Actinomyces naeslundii, Tannerella forsythia, Streptococcus mutans, Streptococcus gordonii, and Candida albicans as well as help in reducing gingivitis and plaque.

This leads us to study about the role they could play probiotics in children and adolescents with oral diseases, and the benefits to be gained from them.
\end{abstract}




\title{
Carcass, Haematological and Serum Biochemical Values of Arbor Acre Broiler Chickens Fed Vary Levels Rumen Liquor Fermented Rice Husk Meal Based Diets
}

\author{
O. J. Alabi ${ }^{1,2, \varpi,\llcorner}$, J. Y. Adama ${ }^{1}$, O. O.A. Fasanya ${ }^{1}$, E. F. Mbajiorgu ${ }^{3}$ and M. David $^{1}$ \\ ${ }^{1}$ Federal University of Technology, Nigeria \\ ${ }^{2}$ University of Limpopo, South Africa \\ ${ }^{3}$ University of the Witwatersrand Johannesburg, South Africa
}

\begin{abstract}
A study was conducted to evaluate the effects of rumen liquor fermented rice husk meal based diets (RLFRHM) on growth, haematological and serum biochemical values of Arbor acre broiler chickens. A total of 200 birds were used. The RLFRHM were included at 0,5,10,15 and 20\%, respectively. The birds were randomly allocated to the five treatments groups, each group had four replicates and there were ten birds per replicate using completely randomized design. The results showed no significant ( $p>0.05)$ differences in live weight, feed intake, feed conversion ratio and mortality. However, the growth rate was influenced by the dietary treatments with chickens on 5\% inclusion level significantly higher than the control (14.96 vs $12.87 \mathrm{~g} / \mathrm{bird} / \mathrm{day})$. Haematological results from this study showed that RLFRHM diets had effect $(\mathrm{p}<0.05)$ only on the mean cell haemoglobin concentration of all the haematological parameters measured with bird on $20 \%$ inclusion having a value of $599.50 \mathrm{~g} / \mathrm{di}$ against the control which had $503.50 \mathrm{~g} / \mathrm{di}$. In the serum electrolyte and biochemical indices evaluated, RLFRHM diets influenced $(\mathrm{p}<0.05)$ the sodium $(147.60 \mathrm{mmol}$ in birds on 5\% inclusion level and 131.57, $136.20 \mathrm{mmol}$ for control and $20 \%$ inclusion levels), chlorine (97.80 in birds on control and 77.36, 67.20, 70.80 and $58.00 \mathrm{mmol}$ for 5, 10, 15 and 20\% inclusion levels) and total bilirubin $(5.70 \mathrm{mg} / \mathrm{dl}$ for $205 \%$ inclusion level against the control $4.25 \mathrm{mg} / \mathrm{dl})$. However, the haematological and biochemical parameters measured were within the normal ranges for chickens. This study showed that RLFRHM based diet at $20 \%$ inclusion level improved growth rate and had no adverse effect on the all haematological and the biochemical parameters measures.
\end{abstract}

\section{Ginkgo Biloba Improves Intestinal Permeability and Liver Function in Rats with Alcohol-induced Liver Injury}

\author{
Xuan $\mathrm{Li}^{\square, \AA}$ and Suhua Pan \\ Nanjing University of Chinese Medicine, Nanjing, China
}

\begin{abstract}
Compound Ginkgo biloba (CGB) is prepared by Ginkgo biloba extract and Rosa roxburghii in a 1:1 proportion based on Traditional Chinese Medicine Theory. Here, its effects on liver function and intestinal permeability are observed using acute and chronic alcohol-induced liver injury animal model. Alcohol was used to induce acute liver injury animal model and LieberDeCarli liquid diet was used to induce chronic liver injury animal model. CGB treatment decreased CYP2E1, NF-Kb, IL-1 $\beta$ and increased Nrf2 expression level in hepatocyte from acute model. CGB significantly reduce the activities of serum ALT, AST, diamine oxidase as well as levels of serum TG, D-lactic acid and plasma endotoxin in chronic liver injury rats. Hepatic steatosis was improved and the damage to intestinal tight junctions was also relieved effectively after CGB administration. Moreover, CGB significantly downregulated the expressions of TNF- $\alpha$, lipopolysaccharide binding protein (LBP), CD14 and TLR4 in the liver and upregulated the expressions of tight junction proteins including ZO-1, occludin and claudin- 1 . In conclusion, CGB alleviated alcohol-induced liver injury and hepatic lipopolysaccharide signaling as well as gut barrier dysfunction through restoring tight junctions.
\end{abstract}




\title{
Poster Abstract
}

\section{Encapsulation Methods of Probiotic Lactic Acid Bacteria}

\author{
Ece Gokmen ${ }^{\varpi, \dot{a}}$ and Dilek Heperkan \\ Istanbul Technical University, Turkey
}

\begin{abstract}
Probiotics are viable microorganisms which are beneficial for human health. Some positive effects are expected in the human metabolism when these viable cells are consumed. The beneficial health effects of probiotics are numerous such as hydrolysis of lactose, decrease in serum cholesterol level, anticarcinogenic property on colon cancer, control of gastrointestinal system. The most common probiotics are bacteria such as Lactobacillus spp. (e.g. L. casei, L. acidophillus), Bifidobacterium spp. and Streptococcus spp. The probiotic microorganisms should be transported by carriers (i.e. food matrix) in order to make microorganisms active in the human metabolism especially in the gastrointestinal system. The effectiveness of the probiotics in the metabolism is associated with concentration of microorganisms present in the food product and their viability should be preserved from the harmful effect of $\mathrm{pH}$ in the gastrointestinal system as well as during storage. Therefore, microencapsulation technique which is relatively a new technique is used for the probiotics. There are several material for encapsulation. Starch, starch derivatives, alginate, gums (e.g. gellan gum, xanthan gum), $\kappa$-carrageenan, cellulose acetate phthalate, chitosan, gelatin, milk proteins and almond gum are materials used as encapsulating materials. In order to produce microcapsules, there are various techniques such as spray drying, freeze drying, air suspension coating, centrifugal extrusion. Additionally, alginate-starch encapsulation, direct compression encapsulation, rennet-gelled protein encapsulation, whey protein gel particles encapsulation, prebiotic encapsulates, alginate-coated gelatin microsphere encapsulation and an interpolymer complex encapsulation in supercritical carbon dioxide are the procedures that are used to encapsulate the probiotic microorganisms. In this presentation different application of encapsulation among lactic acid bacteria will be discussed.
\end{abstract}

\section{Viability of New Potential Probiotic Strains with Anti-inflammatory \& Anti-obesity Effects during Low-fat Yogurt and Cheddar Cheese Making}

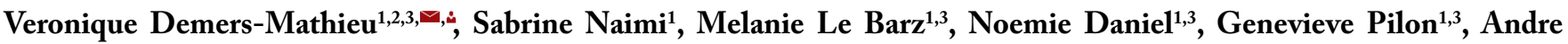 \\ Marette $^{1,3}$,Julie Audy ${ }^{4}$ Emilie Laurin ${ }^{5}$, Ismail Fliss ${ }^{1}$ and Daniel St-Gelais ${ }^{1,2}$
}

${ }^{1}$ Laval University, Canada

${ }^{2}$ Agriculture and Food Canada, Food Research and Development, St-Hyacinthe, Canada

${ }^{3}$ Quebec Heart and Lung Institute (CRIUCPQ), Canada

${ }^{4}$ Agropur Cooperative, Canada

${ }^{5}$ Aliments Utima Inc., Canada

\begin{abstract}
Obesity is associated with chronic low-grade inflammation. Some probiotics (Lactobacillus, Bifidobacterium) have contributed to prevent obesity and inflammation by improving intestinal barrier function with the decrease of lipopolysaccharides (LPS) circulation, by decreasing the liberation of pro-inflammatory metabolites such as cytokine (IL-6, TNF- $\alpha$ ) and nitric oxide (NO), and by increasing the production of anti-inflammatory cytokine (IL-10). No dairy products containing anti-inflammatory and anti-obesity probiotics are currently available in North America. The viability of probiotic bacteria is important during the manufacture and storage of dairy products since cheese $(30 \mathrm{~g})$ and yogurt $(100 \mathrm{~g})$ must contain at least 109 of probiotic strain with health claims, according to the regulation of probiotic foods.

The objective of this study was to determinate anti-inflammatory (in vitro) \& anti-obesity (in vivo) effects of new probiotics and their viability during production and storage of low-fat yogurt and Cheddar cheese. By using 16S genetic identification; specific Q-PCR primers have confirmed lactis subspecies of two Bifidobacterium animal is isolated from infants or adults feces and RpoB primers have validated the species of three Lactobacillus strains isolated from dairy products. Cell model of inflammation and model mice obese with milk as vector were used.

The heat-killed of probiotic strains induced significant decrease in NO production and were able to increase IL-10 production (except L79) in macrophages. The body weight gain of mice fed with high-fat high-sucrose (HFHS) diet supplemented with probiotic milks was significantly reduced comparing to mice fed with HFHS diet and WP milk. Probiotic cells were viable in dairy products after the production and storage of low-fat yogurt and Cheddar cheese as recommended FDA. However, in vitro
\end{abstract}


study suggests that death probiotic cells could play a role in anti-inflammatory effect, whereas in vivo study proposes that viable probiotic cells could have anti-obesity effect.

In the next study, some of these probiotic cheeses and yogurts will be evaluated with model mice obese to determine their anti-inflammatory effect in these products.

\title{
Anti-adipogenic Effect of Kimchi Fermented in Kimchi Refrigerator under Different Storage Conditions
}

\author{
Ki-Bum Park ${ }^{1,2, \varpi, \imath,}$,Yeon-Jeong Moon ${ }^{3}$, P. B. Tirupathi Pichiah ${ }^{3}$, Youn-Soo Cha ${ }^{3}$,Jong-in Jeon ${ }^{2}$ and Suk-Heung Oh ${ }^{1}$ \\ ${ }^{1}$ Woosuk University, Korea \\ ${ }^{2} \mathrm{DAYOU}$-WINLA Inc., Korea \\ ${ }^{3}$ Chonbuk National University, South Korea
}

\begin{abstract}
Obesity, most often defined as a body mass index (BMI) of $\geq 30 \mathrm{~kg} / \mathrm{m}^{2}$ caused by an imbalance between energy intake and expenditure. It is widely recognized as the largest and fastest growing public health problem in the developed and developing world. Obesity results by an increase in adipose tissue mass, resulting from the multiplication of fat cells followed by adipogenesis and increased deposition of intracellular cytoplasmic triglycerides. In this study, we investigated the anti-adipogenic properties of the extracts (KEs) obtained from Kimchi (Korean traditional fermented vegetable) fermented using Kimchi refrigerator under different storage conditions. In order to examine the proliferation of pre-adipocytes, cell growth inhibition was measured over a period of time (initial, $24 \mathrm{~h}$ and $48 \mathrm{~h}$ ) using cell viability assay. The intracellular lipid droplets and triglyceride accumulation in the differentiated adipocytes were also measured. We observed that the KEs significantly lower the contents of cellular triglyceride and formation of intracellular lipid droplets via inhibiting the differentiation of 3T3-L1 adipocytes. These results suggested that KEs have anti-adipogenic effects and may contribute to food industries in developing functional foods based therapies for the prevention of obesity. This research was funded by DAYOU-WINIA Inc.
\end{abstract}

\section{Symptom-free Antibiotic Administration Reduced Mouse Gut Inner Mucus Layer and Bacterial Diversity}

\author{
Ya-Ting Hsu ${ }^{\square, \iota}$, Kai-Chieh Hsu and Han-Chen Ho \\ Tzu-Chi University, Taiwan
}

\begin{abstract}
The intestinal mucus of mouse colon is organized into two layers. The inner layer is devoid of bacteria, while the outer layer is densely colonized by bacteria. Antibiotic is known to disturb gut microbiota, however, whether changes of microbiota associated with mucus layer is less discussed. The aim was to evaluate and visualize the impact of broad spectrum antibiotics on the gut epithelial junctions, mucus layer, and microbial community. Mice were treated with both streptomycin and bacitracin for 7 days. Colon was isolated and divided into proximal, middle, and distal parts. We found that antibiotics-treated mice were physiologically normal: no diarrhea, body weight loss, tight junction dysfunction can be detected. Antibiotics-treated mice showed significantly increased inner mucus layer in proximal and middle colon. Besides, there was a dramatically decreased bacterial number in the outer mucus layers. Interestingly, the thickness of inner mucus layer retuned to control level, and the bacteria re-colonized at the outer mucus layer in antibiotics-treated mice following antibiotic withdrawal for 7 days. By analyzing $16 \mathrm{~S}$ rDNA sequences, we found that major groups of bacteria are not differed in 3 parts of colon: In control mice, Firmicutes dominate the community: over 50\% are Lachnospiraceae and Ruminococcaceae; Lactobacillus and Staphylococcus made up $20-35 \%$ of the community. Surprisingly, antibiotics eliminated most of the gut bacteria, and Turicibacter comprised over $90 \%$ of the survived bacteria. Furthermore, in antibiotic withdrawal mice, microbial community returned to a more complex structure similar to control mice. Although remained different from control, Lachnospiraceae and Ruminococcaceae quickly re-colonized the gut and became the dominant groups of bacteria.
\end{abstract}




\title{
Enhanced Butyrogenic Property of Slow Fermented Corn Arabinoxylans after Oxalic Acid Treatment
}

\author{
Pinthip Rumpagaporn ${ }^{1}$, Thaisa Moro Cantu-Jungles ${ }^{1,2, \varpi,\llcorner}$ and Bruce R. Hamaker ${ }^{1}$ \\ ${ }^{1}$ Purdue University, USA \\ ${ }^{2}$ Universidade Federal do Paraná, Brazil
}

\begin{abstract}
Previous work in our laboratory showed that alkali-solubilized corn arabinoxylan (CAX) had a slow initial, but later complete, human fecal microbiota fermentation. CAX and a still fairly high molecular weight hydrolyzate $(\mathrm{CH})$ were propiogenic, but produced low levels of butyrate. Here, we show that oxalic acid-generated hydrolyzates from CAX, which includes a large xylooligosaccharide and free arabinose fraction, increased and altered short chain fatty acid (SCFA) profiles. Hydrolytic degradation of CAX by acid hydrolysis $\left(0.05 \mathrm{M}\right.$ oxalic acid at $100^{\circ} \mathrm{C}$ for $\left.2 \mathrm{~h}\right)$ and graded ethanol precipitations were used to obtain compounds with different molecular sizes. Each ethanol-precipitated fraction (F0-65\%, 65-75\%, 75-85\%) was mostly lower than $100 \mathrm{kDa}$, and $\mathrm{F}>85 \%$ was composed of monosaccharides and oligosaccharides of DP 2-8. Oxalic acid treatment caused the removal of all single arabinose unit branch chains and some di/trisaccharide branch chains of CAX, and produced lightly substituted and linear xylan backbone fragments, most which were in the oligosaccharide size range. In vitro human fecal fermentation showed all acid-hydrolyzate fractions were initially slower fermented than fructooligosaccharides (FOS), but produced similar or higher amounts of total SCFAs. Butyrate production increased significantly over CAX, while propionate remained relatively high.
\end{abstract}

\section{Characterization and Screening of the Microflora of Lemon Coagulant Soy-Cheese for Preliminary Starter Culture(s)}

\author{
Jemima Omonigho Adepehin ${ }^{1,2, \square}$ \\ ${ }^{1} \mathrm{Federal}$ University of Technology, Nigeria \\ ${ }^{2}$ University of California, Davis, USA
}

\begin{abstract}
Naturally fermented soy-cheese (fermentation time: $12 \mathrm{~h}$ at $37^{\circ} \mathrm{C}$ ) was produced and its microflora was enumerated, isolated and identified. The dominating fermenting microorganisms (lactic acid bacteria) were screened for possible use as starter cultures based on acidification profile and physico-chemical properties of the soy- cheese produced. The total viable cell count of the naturally fermented soy- cheese was 5.30 x $106 \mathrm{cfu} / \mathrm{g}$ and that of the lactic acid bacteria count reported $4.15 \times 105$ $\mathrm{cfu} / \mathrm{g}$. The microflora of the soy-cheese includes; Aerococcus viridians, Micrococcus kristinae, Micrococcus varians, Leuconostoclactis, Lactobacillus fermentum, Lactococcus lactic spp. cremoris, Lactobacillus pentosus, Lactobacillus delbrueckii spp. bulgaricus, Lactobacillus brevis, Escherichia coli, Aeromonas hydrophila, Saccharomyces cerevisiae and Candida sake. The lactic acid bacteria isolates from the naturally fermented soy- cheese were used as single culture to produce soy-cheese. The $\mathrm{pH}$ of the soy-cheese ranges from 5.41 to 5.74 and its protein content ranges from $27.34 \%$ to $51.85 \%$. Among the lactic acid bacteria used as starter culture, the results indicated that Lactobacillus delbrueckii spp. bulgaricus, Lactobacillus pentosus and Leuconostoclactis spp. cremoris yielded the highest protein contents in soy-cheese. Hence, it can be deduced that they hold potential for use as starter cultures and the combination of soy-cheese isolates in mixed culture fermentation trial is recommended.
\end{abstract}

\section{Effect of Red Meat Addition on the Microbiological, Physicochemical and Sensory Properties of Dairy Yoghurt}

\author{
Jawad Al Khalaf ${ }^{1}$, Noemi Gutierrez-Maddox ${ }^{1,2, \square, 2}$ and Nazimah Hamid ${ }^{1,2}$ \\ ${ }^{1}$ School of Applied Sciences, AUT University, New Zealand \\ ${ }^{2}$ Centre for Food Science, AUT University, New Zealand
}

\section{Abstract}

Addition to dairy yoghurt of cooked mince beef was performed to provide additional nutritional benefits to this food product. Yoghurts were manufactured in which whole milk was replaced with meat such that the solids content remained constant at the 
added meat levels of 5\%,7\% and 9\%. At these replacement levels, the production of acidity and the microbiological counts of the yoghurts were unaffected. As the level of meat replacement increased the protein content of the yoghurts increased, while the fat content decreased. The increased meat content was also related to an increase in colour and syneresis of the yoghurt and a decreased viscosity. Sensory analysis revealed that there were significant differences between the control and the $7 \%$ and $9 \%$ meat replacements, but for the 5\% replacement there were no significant differences from the control in overall liking, flavor and odour. Thus, this level of replacement can provide increased nutritional quality while remaining acceptable to the consumer.

\section{Submit Your Next Manuscript}

We are currently accepting submissions from new authors. Read our inaugural issue articles and submit your next manuscript to Journal of Food Chemistry and Nanotechnology (JFCN).

Submit Online: http://www.editorialmanager.com/usg

Citation: Proceedings of the First Probiotics Summit (PBS-2015). J Food Chem Nanotechnol 1(Suppl 1): S1-S16.

Copyright: This is an Open Access article distributed under the terms of the Creative Commons Attribution 4.0 International License (CC-BY) (http://creativecommons.org/licenses/by/4.0/) which permits commercial use, including reproduction, adaptation, and distribution of the article provided the original author and source are credited. Published by United Scientific Group.

Received: September 14, 2015 Accepted: October 08, 2015 Published: October 13, 2015 